\title{
7th European Conference on Atomic Spectroscopy
}

\author{
Grenoble, France, 8-11 July 1975
}

The European Conferences on Atomic Spectroscopy, abbreviated to EGAS Conferences, are by now well established. Initiated during the atomic-spectroscopy symposium in Cain in 1968 and officially started in Paris in 1969, these conferences, due to their success, rapidly increased to become truly European, and EGAS is now a section of the Atomic Physics Division of the European Physical Society.

The EGAS Conferences are in many respects different from other international conferences of today. Their main purpose is to give young physicists a possibility to present and discuss the results of their research at an international meeting ; thus only a few invited papers are presented. This year, J.C. Pebay-Peyroula and his coworkers at Laboratoire de Spectrométrie Physique, Université de Grenoble, organized the EGAS Conference on the Grenoble University campus. About 225 physicists, predominantly from Western Europe attended the meeting, but the increasing number of participants from outside Europe is noteworthy.

The impact of lasers, particularly tunable dye lasers, on atomic spectroscopy was fully recognized at this year's conference. Two of the invited lectures and $\sim 25 \%$ of the nearly 100 contributed papers dealt with the use of lasers. In his lecture on 'Laser Spectroscopy', R. Wallenstein, Stanford University, gave a review of lasers and their applications to atomic-structure studies, touching upon the more recent developments at Stanford, such as UV lasers and Doppler-free, twophoton spectroscopy of hydrogen. Useful objects for Doppler-free, two-photon spectroscopy in a not too distant future will be the inert gases, for which promising results were presented by the group at Laboratoire de Spectrométrie Hertzienne, Paris.

The combination of highly collimated atomic beams and narrow-band tunable dye lasers allows a high-resolution spectroscopy without Doppler broadening. A number of groups applying this technique reported on precise measurements of hyperfine structure and isotope shifts for alkali atoms, inert gases, and rare earths together with lifetime studies performed by means of stepwise excitation by twopulsed dye lasers.

V.S. Letokhov, Institute of Spectroscopy, Moscow, gave an invited talk on spectroscopy in $\gamma$ regions without Doppler broadening with the use of lasers in which the possibility of laser application to nuclear-physics studies was discussed on the basis of experiments performed in Moscow. Two papers clearly demonstrated the potentiality of combining techniques from nuclear and atomic physics to obtain information on nuclear properties from short-lived isotopes. Isotope shifts and hyperfine-structure properties were reported by the Paris group for shortlived $\mathrm{Na}$ isotopes studied by means of high-resolution spectroscopy, and $\mathrm{C}$. Ekström, Isolde-CERN, presented nuclear-spin values for $\mathrm{Au}$ isotopes obtained with an atomic-beam apparatus connected on-line to the isotope separator in which the target is irradiated with protons.

To illustrate the close correspondence between atomic-structure studies performed by means of atomic spectroscopy and the rapidly progressing fields of solid-state spectroscopy and muonic atomic studies, R. Hänsel (Kiel) and J. Duclos (Saclay) gave invited talks on 'Inner-shell Transitions and Synchrotron Radiation' and 'Atomic Physics and Basic Interactions', respectively.

The major part of the contributed papers were presented in sessions with the following titles: Term Analysis, Resonance Fluorescence and Optical Pumping, Beam-Foil Spectroscopy, Hyperfine Structure, Atomic Transition Probabilities, and Atomic Collision Cross Sections. The potentiality of the experimental and theoretical methods used in term analysis today was demonstrated by a series of studies including transition elements and uranium, which have spectra containing more than 100,000 spectral lines in the visible region, and by the success of multi-configuration Hartree-Fock calculations for the solving of problems such as the $\mathrm{sp}^{6}$ term position in the halogens.

Singlet-triplet separations, fine and hyperfine structure, lifetimes and transition probabilities for atomic and ionic systems were the physical properties under discussion in the level-crossing, beam-foil, hyperfine-structure, and transition-probability sections. At present there is a clear distinction between the light atomic systems $(Z \leqslant 10)$, for which there exist advanced theoretical predictions and where highprecision experimental data for further development are necessary, and the more complex heavier atomic systems for which the theoretical studies are of semi-quantitative nature.

This was exemplified at the conference, where experimental and theoretical studies of the hyperfine interactions of the lowest ${ }^{2} \mathrm{~S}$ and ${ }^{2} \mathrm{P}$ states in $\mathrm{Be}^{+}$were presented with the manybody calculated values being essentially in agreement with experimental values obtained by optical pumping or fast-beam level-crossing techniques. Further development of many-body calculations to higher excited states in 3-electron systems (i.e., doubly excited states) or to 4-5-electron systems are justified since the quantum-beat technique after beam-foil excitation now yields experimental results with sufficient accuracy to warrant detailed comparison.

Although the progress in theoretical calculations of heavier atomic systems is still limited, there is a clear indication that considerable improvements are now on the way. Accurate lifetime calculations of excited levels in inertgas ions were an example presented at the conference, the theoretical values reproducing the most recent experimental values in a satisfactory manner. The main experimental contributions dealing with lifetime studies, oscillator strengths, Landé g factors, hyperfine structure, and isotope shifts for heavier systems were concentrated to the inert gases, $\mathrm{Zn}, \mathrm{Cd}, \mathrm{Hg}$, and the rare earths.

The scientific standard of this conference shows promise for the next EGAS Conference, which will be held in Oxford in 1976

Three new members, J. Bauche, Orsay, M. D. Kunisz, Cracow, and R. Winkler, Berlin-West, were elected to the new EGAS Board, where D.N. Stacy, Oxford, will replace A. Steudel, Hannover, as Chairman.

\section{T. Andersen, Aarhus}

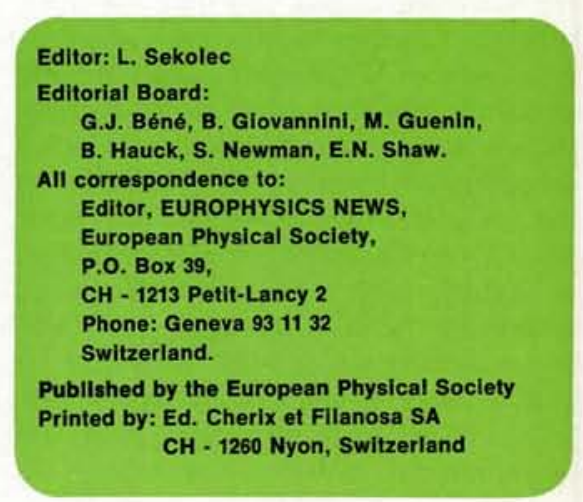

